geringen Teil wieder rückadsorbiert wird, und zwar bei geringen Konzentrationen entsprechend der Freundlichschen Adsorptionsisotherme relativ mehr als bei höheren. Man muß also innerhalb dieses Bereiches die Auswertung an Hand der Eichkurve vornehmen.

Die Gegenwart von Natrium, Kalium, Magnesium und Calcium beeinträchtigt die Ergebnisse nicht, wenn diese Ionen in gleicher Größenordnung wie das Fluor auf den Zirkonphosphatfleck aufgegeben werden. Eisen hingegen maskiert das Fluor, so daß die Phosphorsäure nicht quantitativ freigesetzt wird.
Versuche, die Umsetzung in Lösung mit größeren Volumina der zu untersuchenden Fluorlösung vorzunehmen und die Fluormenge nach Abzentrifugieren des Mischniederschlages aus Zirkonfluorid und Zirkonphosphat durch Messen der in der flüssigen Phase auftretenden Phosphorsäure zu bestimmen, scheiterten, weil stets einige Prozent des vorliegenden Zirkonphosphats peptisiert werden. Der peptisierte Anteil ließ sich auch durch Filtration mit einem Ultrafilter nicht abtrennen. Im Chromatogramm wandert er jedoch nicht mit dem Lösungsmittel mit, so daß er hier nicht stört.

\title{
Über östrogen wirksame Thiole
}

\author{
Von Anneliese Hillmann-Elies \\ Aus dem Chemischen Laboratorium der Medizinischen Univ.-Klinik Tübingen \\ (Dir. Prof. Dr. B e n n h old) \\ (Z. Naturforschg. 10 b, 442-444 [1955]; eingegangen am 14. April 1955)
}

\begin{abstract}
Es wird die Synthese des 3.4-Bis-[4-acetylmercaptophenyl]-hexans beschrieben und über die Wirkung im Allen-Doisy-Test berichtet.
\end{abstract}

Z u den östrogenen wirksamsten Substanzen gehören außer den natürlichen Follikelhormonen die Bisdehydroisynolsäure, die Doisynolsäure, 3.4Bis-[4-oxyphenyl]-hexan-Abkömmlinge und Triphenylhalogenäthane. In neuerer Zeit haben radioaktiv markierte Östrogene zum Studium des Stoffwechsels dieser Stoffe Bedeutung gewonnen. Dazu wurden die bisher bekannten Verbindungen mit ${ }^{14} \mathrm{C}$ (Östron ${ }^{1}$, 17-Methylöstradiol ${ }^{2}$, Stilböstrol ${ }^{3}$ ) oder Tritium (Hexöstrol ${ }^{4}$ ) markiert oder die radioaktive Kennzeichnung durch Einführung von ${ }^{131} \mathrm{~J}$ oder ${ }^{82} \mathrm{Br}$ (Jodöstradiole $^{5}$, Jodöstron, Dijodhexöstrol, Jodbisdehydrodoisynolsäure ${ }^{6}$, 7.8-Dibromöstron ${ }^{7}$, Bromtriphenyläthan ${ }^{8}$ ) erreicht. Die Verwendung der ${ }^{14} \mathrm{C}$-markierten Östrogene ist auf Grund ihrer geringen spezifischen Aktivität nur für Ausscheidungsversuche im Tierexperiment geeignet, während die ${ }^{131} \mathrm{~J}$-markier-

1 R. D. H. H e a r d, C. P. L e blo nd u. a., Rev. can. Biol. 10, 73 [1951].

2 E. A. D o is y u. a., J. biol. Chemistry 202, 27 [1953].

3 D. J. H a n a han u. a., Fed. Proc. 10, 194 [1951].

4 R. F. G la s c o ck u. L. R. Re in ius, Isotop. Conference, S. 262, Oxford 1954.

5 R. D. H. H e a r d, C. P. L e blond u. I. S a f f r a n, J. biol. Chemistry 177, 247 [1949].

6 Eigene Befunde.

7 G. H. Twombly, L. M c Clintock u. M. En g e lm a n n, Amer. J. Obstetr. Gynecol. 56, 260 [1948]. ten Substanzen zwar die Messung der Verteilung im Tierorganismus erlauben, jedoch enzymatisch verschieden leicht dejodiert werden. Daher erscheint es sinnvoll, Thiole zu synthetisieren, die die pharmakologische Wirkung des Follikelhormons besitzen und die damit die Möglichkeit zur Untersuchung mit ${ }^{35} \mathrm{~S}$ markierten Östrogenen bieten. Das Schwefelisotop ${ }^{35} \mathrm{~S}$ ist infolge seiner günstigen Halbwertszeit (88 d) und der besseren Wirkungsausbeute der $\beta$-Strahlen bei der Messung gegenüber der geringen Wirkungsausbeute der $\gamma$-Strahlen des ${ }^{131} \mathrm{~J}$ besonders gut für Isotopenversuche geeignet.

Es erschien ferner von Interesse, festzustellen, welchen Einfluß die Einführung des Schwefels an Stelle von Sauerstoff auf die biologische Aktivität von Steroiden und Steroidanalogen ausübt. An Thioderivaten von Steroiden sind bisher bekannt: 21-Thiolacetate von Desoxycorticosteron ${ }^{9}$ und 3-Thioderivate von Cholesterin ${ }^{10}$, Provitamin D ${ }^{11}$, Dehydroepiandro-

8 P. Daudel, M. B erger, N. P. Bu u-Hoi u. A. La c a s s a g n e, Experientia [Basel] 2, 107 [1946].

9 C. D jerassi u. A. L. Nus s baum, J. Amer. Soc. 75, 3700 [1953].

10 T. W a gne r-J a uregg u. T. Lennatz, Ber. dtsch. chem. Ges. 74, 27 [1941].

11 J. S trating u. H. J. B a cker, Recueil Trav. chim. des Pays-Bas 69, 909 [1950]. 
steron ${ }^{12}$. Úber eine biologische Wirkung dieser Stoffe sind keine Angaben gemacht.

Als Modellsubstanz für weitere Untersuchungen wurde die Synthese des 3.4-Bis-[4-mercaptophenyl]hexans durchgeführt. Da die phenolische Gruppe des 3.4-Bis-[4-oxyphenyl]-hexans (Hexöstrol) sich nicht durch Schwefel ersetzen ließ und die Pinakonreduktion des durch Friedel-Craffts-Reaktion leicht zugänglichen $p$-Acetaminopropiophenons zu 3.4-Bis-[4-acetaminophenyl]-hexandiol-(3.4) unter verschiedenen Bedingungen nur in schlechter Ausbeute gelang, wurde die Synthese in folgender Weise durchgeführt: tung mit Essigsäureanhydrid-Acetylchlorid und anschließende Hydrierung mit $\mathrm{Pd} / \mathrm{BaSO}_{4}$ wurden daraus die meso- und die D,L-Form des 3.4-Diphenylhexans ${ }^{14}$ (III) erhalten. Aus dem Gemisch wurde durch Behandeln mit Methanol eine kristalline neben einer öligen Fraktion erhalten, über deren sterische Zuordnung keine Hinweise vorlagen. Die nachfolgenden Reaktionen - Überführung in das 3.4-Bis-[4oxyphenyl]-hexan - brachten den Nachweis, daß die kristalline Verbindung die meso-Form war. Die Dinitrierung des 3.4-Diphenylhexans (IV) ließ sich nur mit Bortrifluorid-Eisessig und der berechneten Menge<smiles>CCC(O)(c1ccccc1)C(O)(CC)c1ccc(I)cc1</smiles>

I<smiles>CCCC(CC)(c1ccccc1)c1ccc(C)cc1</smiles><smiles>CCC(c1ccccc1)C(CC)c1ccc(I)cc1</smiles>

III<smiles>CCC(c1ccc([N+](=O)[O-])cc1)C(CC)c1ccc([N+](=O)[O-])cc1</smiles>

IV<smiles>CCC(c1ccc(N)cc1)C(CC)c1ccc(N)cc1</smiles>

V<smiles>CCC(c1ccc(S)cc1)C(CC)c1ccc(S)cc1</smiles>

Aus Propiophenon wurde durch Reduktion mit Aluminiumamalgam das bekannte 3.4-Diphenylhexandiol-(3.4) ${ }^{13}$ (I) hergestellt. Durch Wasserabspal-

$12 \mathrm{~S}$. B ernstein u. K. S a x, J. org. Chemistry 16, 679 [1951].

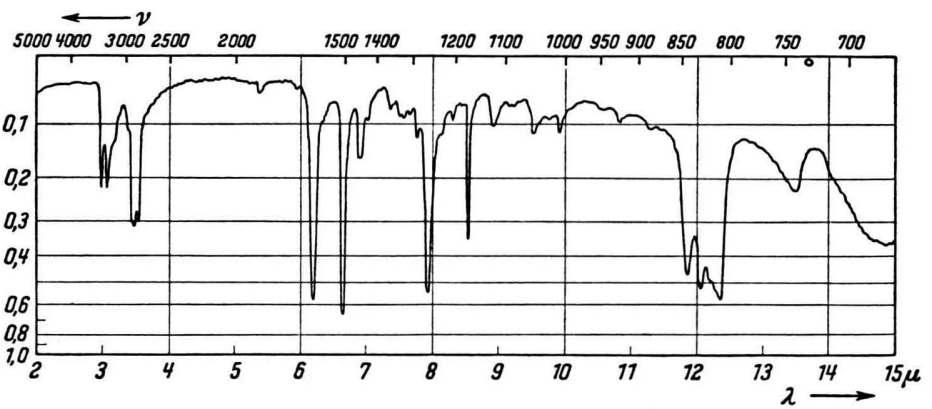

Abb. 1. IR-Spektrum des 3.4-Bis-[4-aminophenyl]-hexans.

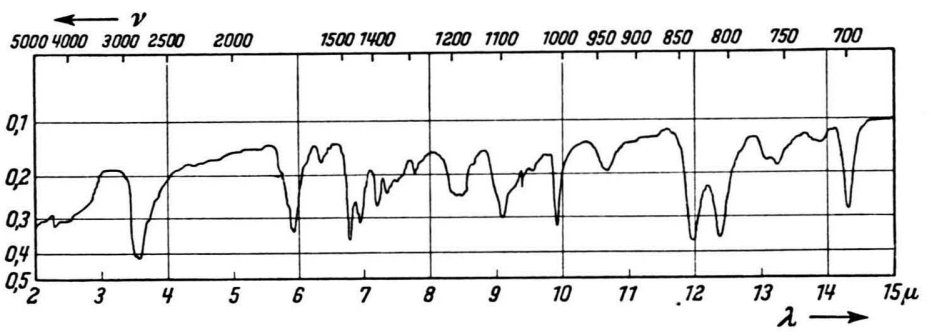

Abb. 2. IR-Spektrum des 3.4-Bis-[4-acetylmercaptophenyl]-hexans.

wasserfreier Salpetersäure erreichen. Durch Hydrierung der Nitrogruppen mit Raney-Nickel wurde das 3.4-Bis-[4-aminophenyl]-hexan (V) hergestellt, dessen IR-Spektrum (Abb. 1) durch die Absorption bei 12,05 bis $12,35 \mu$ eindeutig die para-Stellung der Aminogruppe erkennen ließ. Durch Diazotieren und Verkochen des Amins in der üblichen Weise wurde ein 3.4Bis-[4-oxyphenyl]-hexan erhalten, das ebenso wie das daraus hergestellte Diacetat und Benzoat mit dem bekannten 3.4-Bis-[4-oxyphenyl]-hexan (Hexöstrol), dessen meso-Form ${ }^{14 a}$ gesichert ist, und seinen entsprechenden Derivaten übereinstimmte. Zur Synthese des

13 R. S t e r n, Mh. Chem. 26, 1559 [1905].

14 S. Le p in u. R e i c h, C 1916 I, 757.

14a F. von Wessely u. Willeba, Ber. dtsch. chem. Ges. 74, 777 [1941]. 
Thiols wurde das Amin diazotiert, mit Kaliumxanthogenat umgesetzt und der Xanthogensäureester mit Lithium-Aluminiumhydrid reduziert. Das 3.4-Bis-[4mercaptophenyl]-hexan (VI), das den charakteristischen Thiophenolgeruch zeigte, wurde nicht kristallin erhalten. Durch schonende Acylierung konnte das schwer kristallisierende Diacetat hergestellt werden, dessen IR-Spektrum (Abb. 2) die für Thiolester charakteristische Bande bei 5,93 $\mu$ zeigt.

Die Testierung im Allen-Doisy-Test an der kastrierten Ratte hatte folgendes Ergebnis:

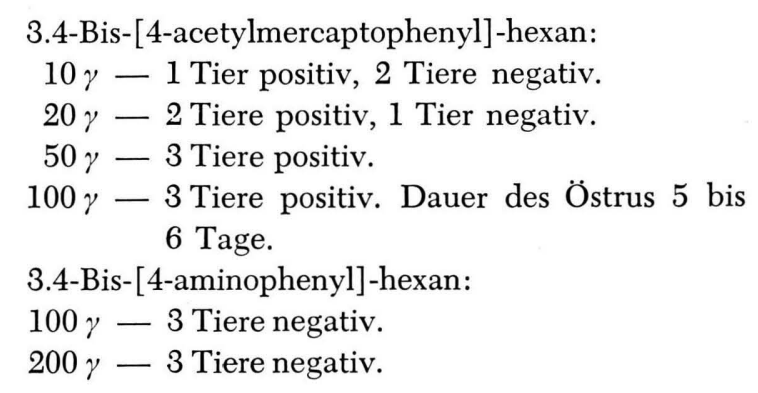

Das 3.4-Bis-[4-aminophenyl]-hexan hemmte in Dosen von $1 \mathrm{mg}$ pro die subcutan die Wirkung des Hexöstrols im Allen-Doisy-Test nicht.

\section{Experimenteller Teil}

3.4 - D i p h en ylh ex andiol $(3,4)(\mathrm{I})$

$20 \mathrm{~g}$ Propiophenon wurden in $200 \mathrm{ccm}$ feuchtem ÄtherEssigester $(1: 1)$ 2-mal mit je $4 \mathrm{~g}$ amalgamiertem Aluminiumgrieß reduziert. Das erhaltene öl wurde mit Petroläther behandelt; nach längerer Kühlung auf $-15^{\circ} \mathrm{kri}-$ stallisiert das Diol aus. Ausbeute: 18-22\%. Schmp. 138 bis $139^{\circ}$.

\section{4 - 3.4 - Diphenylhex a dien (I I)}

$5 \mathrm{~g}$ Diol wurden $2 \mathrm{Stdn}$. auf dem Wasserbad mit $40 \mathrm{ccm}$ Essigsäureanhydrid-Acetylchlorid (1:1) erhitzt. Ausbeute: 65\%. Schmp. $103^{\circ}$ (aus Methanol).

\section{4 - D i p he nyl-hex an (I I I)}

Das Hexadien wurde mit $\mathrm{Pd}$ auf $\mathrm{BaSO}_{4}$ in ÄthanolEssigester $(1: 1)$ hydriert. Ausbeute: $45 \%$. Schmp. $93^{\circ}$ (aus Methanol).

\section{4 - B is - [4 - nitro-phenyl] - he xan (IV)}

$\mathrm{Zu}$ einer Lösung von $2 \mathrm{~g}$ Diphenylhexan in $10 \mathrm{ccm}$ Eisessig, der frisch mit Bortrifluorid gesättigt war, wurden Iangsam unter Rühren $0,46 \mathrm{ccm}$ stickoxyd-freie Salpetersäure $(d 1,495)$ gegeben. Die Temperatur darf $35^{\circ}$ nicht übersteigen. Nach 30-minütigem Stehen wurde die Mischung in Eiswasser gegossen. Die abfiltrierten Kristalle wurden mit warmem Methanol digeriert und aus Aceton umkristallisiert. Ausbeute: $60 \%$. Schmp. 176-177 .

$$
\begin{array}{llll}
\mathrm{C}_{18} \mathrm{H}_{20} \mathrm{O}_{4} \mathrm{~N}_{2} & \text { Ber. } & \mathrm{N} & 8,52 . \\
& \text { Gef. } & \mathrm{N} & 8,70 .
\end{array}
$$

\section{4 - B is - [2.4 - dinitrophenyl] - hexan}

$1 \mathrm{~g}$ Diphenylhexan wurde vorsichtig in $8 \mathrm{ccm}$ Nitriersäure [konz. Schwefelsäure : Salpetersäure $(d$ 1,49), $3: 1$ ] bei $5^{\circ}$ eingetragen und 1 Stde. bei Zimmertemperatur stehen gelassen. Nach Zersetzen mit Eiswasser wurde das in allen organischen Lösungsmitteln schwer lösliche 3.4Bis-[2.4-dinitrophenyl]-hexan in $80-90 \%$ Ausbeute isoliert. Schmp. $222^{\circ}$.

$$
\begin{array}{llll}
\mathrm{C}_{18} \mathrm{H}_{18} \mathrm{O}_{8} \mathrm{~N}_{4} & \text { Ber. } & \mathrm{N} & 13,39 . \\
& \text { Gef. } & \mathrm{N} & 13,27 .
\end{array}
$$

3.4 - B is - [4-acetylmercaptophenyl] he $x$ a n $(V)$

Dinitrodiphenylhexan wurde in Tetrahydrofuran mit Raney-Nickel unter normalen Bedingungen reduziert. Das Amin wurde aus Essigester mit Petroläther umgefällt. Ausbeute 95\%. Schmp. $144^{\circ}$.

$$
\begin{array}{llll}
\mathrm{C}_{18} \mathrm{H}_{24} \mathrm{~N}_{2} & \text { Ber. } & \mathrm{N} & 10,44 . \\
& \text { Gef. } & \mathrm{N} & 10,41 .
\end{array}
$$

Das mit Pyridin und Essigsäureanhydrid hergestellte Diacetat war schwer löslich in Methanol, Äthanol und Aceton. Schmp. $275^{\circ}$.

$$
\begin{array}{llll}
\mathrm{C}_{22} \mathrm{H}_{28} \mathrm{O}_{2} \mathrm{~N}_{2} & \text { Ber. } & \mathrm{N} & 7,95 . \\
& \text { Gef. } & \mathrm{N} & 8,02 .
\end{array}
$$

3.4 - B is - [4-mercaptophen yl] he $\mathrm{x}$ an (VI)

$2 \mathrm{~g}$ Amin wurden bei $5^{\circ}$ in $7 \mathrm{ccm} 25$-proz. Salzsäure mit $1,05 \mathrm{~g}$ Natriumnitrit diazotiert. $\mathrm{Zu}$ der Diazoniumsalzlösung wurden langsam $6 \mathrm{ccm}$ einer 50-proz. kalten Kaliumxanthogenatlösung gegeben und das Gemisch langsam auf $60^{\circ}$ bis zur Beendigung der Stickstoffentwicklung erwärmt. Das entstandene Xanthogenat wurde mit Benzol extrahiert und ohne Reinigung weiterverarbeitet. Die rohe Xanthogensäure-Verbindung ist ein zähes Öl, das in Methanol und Äthanol schwer und in Petroläther leicht löslich ist. Das Xanthogenat wurde in trockenem Äther mit $800 \mathrm{mg}$ Lithiumaluminiumhydrid reduziert. Nach der Zersetzung mit Essigester und Säure wurde die entstandene Thiophenol-Verbindung mit $n-\mathrm{NaOH}$ aus der ätherischen Lösung extrahiert und mit Essigsäure ausgefällt. Das 3.4-Bis-[4-mercaptophenyl]-hexan ist ölig, schwer löslich in Methanol, Äthanol und leicht löslich in Petroläther. - Das Dithiol wurde mit Pyridin-Essigsäureanhydrid durch 12-stdg. Stehen bei Zimmertemperatur acetyliert. Das Diacetat, das als zähes, helles Öl isoliert wurde und schlecht kristallisiert, wurde durch Umfällen aus Benzol-Methanol gereinigt. Das Diacetat ist schwer löslich in Methanol, Äthanol, leicht löslich in Aceton, Benzol, Essigester, Petroläther. Schmp. 102-106 ${ }^{\circ}$. Ausbeute: 65-70\%.

$$
\begin{array}{llll}
\mathrm{C}_{22} \mathrm{H}_{26} \mathrm{O}_{2} \mathrm{~S}_{2} & \text { Ber. } & \mathrm{S} & 16,58 . \\
& \text { Gef. } & \text { S } & 16,37 .
\end{array}
$$

Für die IR-Spektren danke ich Herrn Dr. E. Bi eckert (Max-Planck-Institut für Biochemie, Tübingen) und für die Unterstützung bei der biologischen Testierung Frl. Dipl.-Biol. B. Ke il. Der De ut s chen Forschungsg e meinschaft danke ich für ihre Unterstützung. 